\title{
News from Internal and Emergency Medicine
}

\section{Domenico Prisco}

Published online: 15 January 2015

(C) SIMI 2015

\section{Dear Readers,}

Internal and Emergency Medicine (IEM) has completed its 9th year of publication and this is my 6th year as Editor-inChief.

One year ago, I wrote that we had the opportunity to publish more papers, especially original contributions, due to the increase from 6 to 8 issues published per year, so decreasing the time the accepted papers stay in an "online first" state. We have reached the stated goal of having no paper in the "online first" state for more than 10-12 months. At the moment, only papers accepted in 2014 are in the queue to be published in regular issues.

The section of Emergency Medicine, has a new chairman, doctor Bruce Adams from San Antonio (TX) (already a valuable associate editor of IEM for several years), whereas the co-editor Peter Rosen, will continue to serve by selecting Medical Illustrations, and will help us with his editing experience.

Dr. Adams is Chair of the Department of Emergency Medicine at the University of Texas, San Antonio. He served in the US Army for over twenty years, after training there in Emergency and Internal Medicine. Emergency Medicine section continues to be more and more active, and receives an ever increasing number of submissions from all over the world.

The HTA and Clinical Evidence section has been modified. The new deputy editors Giorgio Costantino and Giovanni Casazza have done tremendous work to improve the section with new ideas and new coworkers. In 2015 you will see many papers in this section.

I am happy to report that our journal has an ever increasing number of readers internationally as well as within Italy and Europe. We are very happy with the quality of new articles that we are receiving, and feel that our goals for publication are being met.

Thanks to all active coworkers in this adventure!

We hope you continue to enjoy and support our journal, and wish you a wonderful 2015!

Domenico Prisco Editor-in-Chief Internal and Emergency Medicine

D. Prisco $(\square)$ 